\title{
Real-time monitoring of DNA immobilization and detection of DNA polymerase activity by a microfluidic nanoplasmonic platform
}

\author{
Johanna Roether ${ }^{\mathrm{a}, \mathrm{b}}$, Kang-Yu Chü ${ }^{\mathrm{a}}$, Norbert Willenbacher ${ }^{\mathrm{b}}$, Amy Q. Shen ${ }^{\mathrm{a}}$, Nikhil Bhalla, ${ }^{\mathrm{a}, \mathrm{c}, *}$

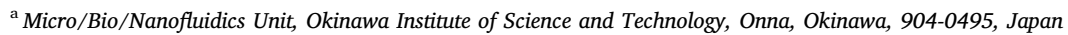 \\ ${ }^{\mathrm{b}}$ Institute of Mechanical Process Engineering and Mechanics, Applied Mechanics Group, Karlsruhe Institute of Technology, 76137, Karlsruhe, Germany \\ ${ }^{\mathrm{c}}$ Nanotechnology and Integrated Bioengineering Centre (NIBEC), School of Engineering, Ulster University, Jordanstown, Shore Road, BT37 0QB, Northern Ireland, United \\ Kingdom
}

A R T I C L E I N F O

Keywords:

LSPR

Microfluidic biosensor

DNA polymerase

Self-assembled-monolayers (SAM)

\begin{abstract}
A B S T R A C T
DNA polymerase catalyzes the replication of DNA, one of the key steps in cell division. The control and understanding of this reaction owns great potential for the fundamental study of DNA-enzyme interactions. In this context, we developed a label-free microfluidic biosensor platform based on the principle of localized surface plasmon resonance (LSPR) to detect the DNA-polymerase reaction in real-time. Our microfluidic LSPR chip integrates a polydimethylsiloxane (PDMS) channel bonded with a nanoplasmonic substrate, which consists of densely packed mushroom-like nanostructures with silicon dioxide stems $(\sim 40 \mathrm{~nm})$ and gold caps $(\sim 22 \mathrm{~nm})$, with an average spacing of $19 \mathrm{~nm}$. The LSPR chip was functionalized with single-stranded DNA (ssDNA) template (T30), spaced with hexanedithiol (HDT) in a molar ratio of 1:1. The DNA primer (P8) was then attached to T30, and the second strand was subsequently elongated by DNA polymerase assembling nucleotides from the surrounding fluid. All reaction steps were detected in-situ inside the microfluidic LSPR chip, at room temperature, in real-time, and label-free. In addition, the sensor response was successfully correlated with the amount of DNA and HDT molecules immobilized on the LSPR sensor surface. Our platform represents a benchmark in developing microfluidic LSPR chips for DNA-enzyme interactions, further driving innovations in biosensing technologies.
\end{abstract}

\section{Introduction}

DNA polymerization, mediated by the enzyme polymerase, assem bles nucleotides along a single stranded DNA, using the latter as a template. This reaction is one of the key steps in the replication of DNA of all types of cells and organisms. Therefore monitoring a DNA poly merase reaction in real time is important in many applications. For example, it is crucial to monitor all reaction steps such as primer binding, enzyme binding, elongation along the template, and the re lease of the enzyme (see Fig. $1 \mathrm{a} \mathrm{c}$ ) for diagnosis and pharmaceutical drug testing. To meet the demand of real time monitoring, some labeled sensing approaches have been developed to detect DNA polymerase activity, which includes discontinuous radio labeled (Benkovic and Cameron, 1995), direct and indirect fluorescence (Shapiro et al., 2005; Seville et al., 1996; Griep, 1995; Ronaghi, 2001), and particle labeled (Sannomiya et al., 2008) assays at bulk and single molecule level. Most of these methods are either time consuming, laborious, cost inefficient or require the usage of toxic chemical reagents (e.g., radioactive tags/ labels).

Among label free methods, quartz crystal microbalance (QCM) serves as a simple and powerful tool for real time measurements (Matsuno et al., 2001), but the measurement response is sensitive to changes in the bulk solution, therefore the signal leads to an over estimation of the number of bound biomolecules (Bingen et al., 2008). The use of localized surface plasmon resonance (LSPR) techniques has recently emerged as an important label free sensing technique: it is an optical phenomenon that causes a collective oscillation of valence electrons and subsequent absorption within the ultraviolet visible (UV Vis) band of the light spectrum, due to interactions between the incident photons and the conduction band of a noble metal nanos tructure (Anker et al., 2010; Hammond et al., 2014; Bhalla et al., 2018a). LSPR is sensitive to the local refractive index around the na nostructures to enable the detection of biomolecule binding events (Mayer and Hafner, 2011). The short decay length of the electro magnetic field in localized surface plasmons makes LSPR relatively insensitive to the bulk effects, thus reducing the sensitivity response to

\footnotetext{
"Corresponding author. Nanotechnology and Integrated Bioengineering Centre (NIBEC), School of Engineering, Ulster University, Jordanstown, Shore Road, BT37 OQB, Northern Ireland, United Kingdom.

E-mail addresses: amy.shen@oist.jp (A.Q. Shen),n.bhalla@ulster.ac.uk (N. Bhalla).
} 


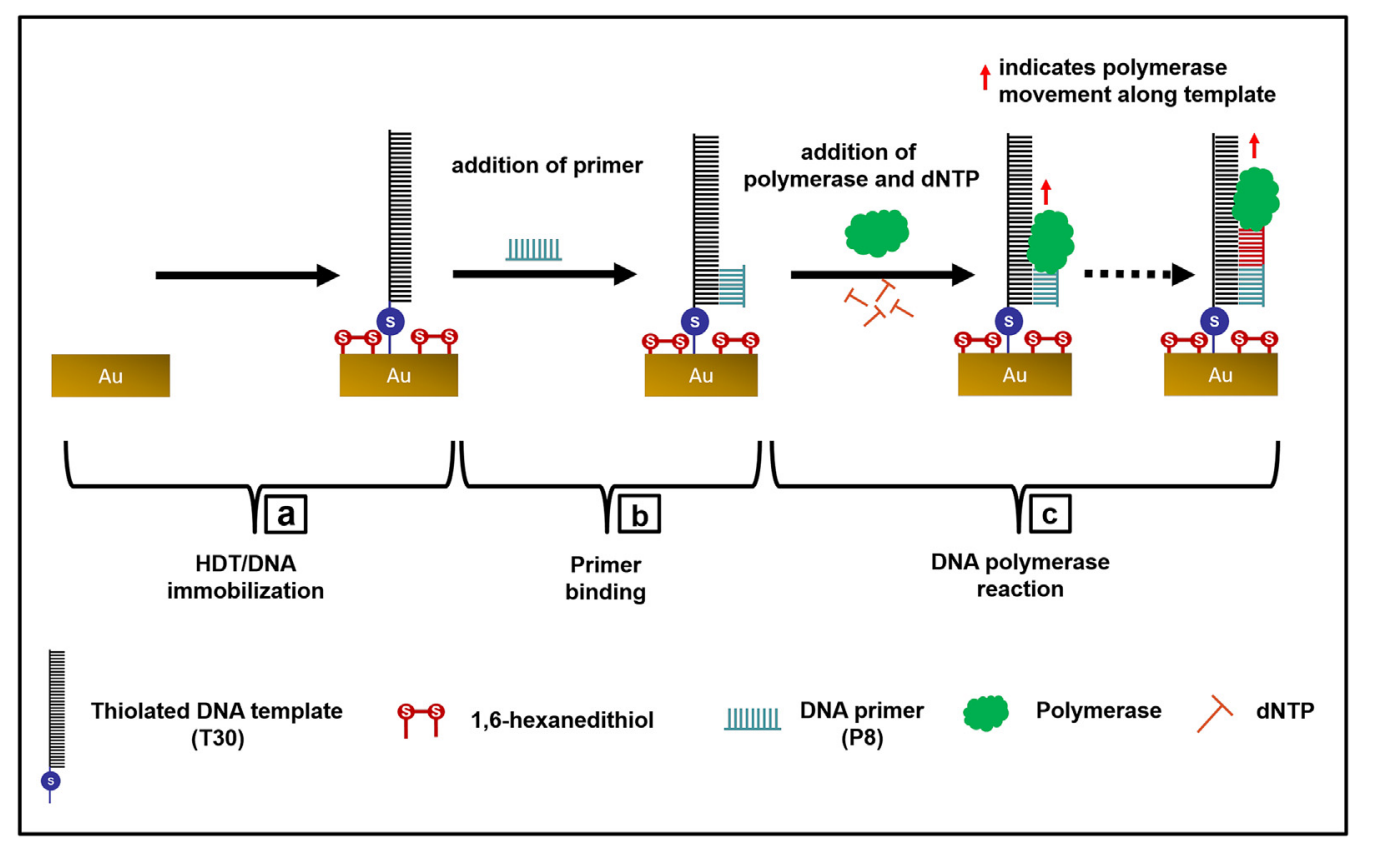

Fig. 1. Reaction scheme on a gold (Au) LSPR substrate, involving (a) an immobilized ssDNA template (T30) with HDT; (b) addition of primer sequence P8, and (c) Klenow fragment of DNApolymerase along with dNTPs. Polymerase catalyzes the formation of the complementary DNA strand by assembling dNTPs from the surrounding media.

the interference from the bulk solution's refractive index (Szunerits and Boukherroub, 2012).

LSPR biosensors have achieved the detection of bio/chemical pro cesses involving DNA, proteins, biomarkers, enzymes, food borne pa thogens, heavy metals, microbial biofilms and even living eukaryotic cells (Bhalla et al. (2018b)). In reference to DNA based sensing, various LSPR biosensors have been successfully implemented to measure DNA hybridization. In particular, chip based (Huang et al., 2012; Soares et al., 2014; Park et al., 2009; Endo et al., 2005) and nanoparticle based (Schneider et al., 2013) approaches have been used for end point analysis of DNA hybridization, serving as efficient alternatives to con ventional polymerase chain reaction (PCR) procedures, enabling highly sensitive quantification of DNA concentrations in solution (Kaye et al., 2017). Kim et al. (2017) and Baaske et al. (2014) recently employed nanorods with whispering gallery modes in microcavities for the de tection of DNA/DNA polymerase interactions and conformational changes at a single molecular level. A combined setup of LSPR and electrochemical impedance spectroscopy has also been used for DNA sensing applications (Cheng et al., 2014).

The sensitivity of LSPR based biosensors can be potentially in creased by integrating it with microfluidics. This is because the mi crofluidic systems provide precise control of the fluid flow, reduce sample volumes, avoid evaporation and enhance the mixing rate of different reagents which often lead to an increase in the sensitivity of biomolecule detection, when integrated with biosensing technologies (Luka et al., 2015). In addition, reactions involving multiple fluid processing steps can be controlled in an automated manner inside a microfluidic chip, thereby avoiding potential measurement errors re sulting from user to user discrepancy. The coupling of microfluidics and biosensors also introduces features such as portability, disposability, and multiplexed analysis of various analytes in a single device. Most importantly, real time measurements can be realized by taking ad vantage of the high surface specificity the LSPR technique for sensing applications (Oh et al., 2014; Aćimović et al., 2014). For instance Oh et al. developed an integrated nanoplasmonic microfluidic chip to de tect cell secreted tumor necrosis factor (TNF) $\alpha$ cytokines in clinical blood samples (Oh et al., 2014) and to detect cancer markers in serum (Aćimović et al., 2014). Touahir et al. (2010) proposed a microfluidic DNA sensing approach based on metal nanostructure enhanced fluor escence, but this requires fluorescence labeling of the DNA probes. More recently, Haber et al. were able to monitor DNA hybridization in real time by combining sensor chips with silver nanoprism structures with a microfluidic setup in a label free manner (Haber et al., 2017). However, to our knowledge, no work on LSPR detection of DNA poly merase reaction in real time has been reported in literature.

Our work successfully demonstrates, for the first time, a LSPR mi crofluidic chip to detect the immobilization of single stranded DNA (ssDNA) mixed with spacer molecules (1 Hexadecanethiol, HDT) on gold nanostructures via thiol chemistry and subsequently detect their interaction with DNA polymerase enzyme in real time at room tem perature. Our LSPR microfluidic platform is superior in distinguishing each step in the polymerase reaction. For instance, we show that events involving binding of small molecules such as the DNA primer (P8) and nucleotides can easily be detected by our LSPR microfluidic chip in real time, in contrast to bulk sensors such as QCM. We also show reduced non specific binding and clear distinction of the polymerase reaction inside the LSPR microfluidic platform in real time, when compared to the traditional LSPR measurements without using microfluidics. Our developed LSPR microfluidic platform may provide a good benchmark sensing platform for DNA based molecular diagnostics.

\section{Materials and methods}

\subsection{DNA immobilization on LSPR substrates}

Thiolated DNA template T30 (S 5'GACGCTAGGATCTGACTGCGCC TCCTCCAT 3 (Hokkaido Gene Design, Japan) was dissolved in TE buffer (100 mM TRIS/10 mM EDTA, pH8), blended in a ratio of 1:1 with the reduction buffer $(0.12 \mathrm{M}$ of Di thiothreitol (DTT): $0.5 \mathrm{M}$ of Phosphate buffered saline (PBS) $=2: 1$ ) and henceforth the reduction of T30 took place at room temperature within $6 \mathrm{~h}$. The DNA was then de salted and the resulting DNA concentration in the TE buffer was mea sured to be $0.66 \mu \mathrm{M}$ (nanodrop fluorometer, Thermo Fisher, Japan). The thiolated DNA was then conjugated on the clean gold based substrates (gold nanostructured LSPR substrates, gold nanostructured LSPR sub strate integrated with microfluidics, and substrates for QCM D) using HDT as a spacer molecule to avoid the steric hindrance, see Fig. 1 (step a). The reaction solution containing $0.45 \mu \mathrm{M}$ DNA and $0.45 \mu \mathrm{M}$ HDT in TE buffer, was deposited on the substrates or pumped through the microfluidic chips to initiate the immobilization within $16 \mathrm{~h}$, all per formed at room temperature. After the immobilization, the functiona lized substrates were washed three times for $15 \mathrm{~min}$ with $1 \times$ PBS. 


\subsection{In vitro DNA polymerase reaction}

The functionalized chips were impinged with primer solution, Fig. 1 (step b), (0.1 $\mu \mathrm{M}$ primer P8 (5 ATGGAGGA 3, Invitrogen), $0.5 \mu \mathrm{M}$ dNTPs (Taraka Bio Inc., Japan), diluted in polymerase reaction buffer (New England Biolabs, NEB), prepared according to manufacturer's manual. The primer binding was carried out for $15 \mathrm{~min}$. After following threefold PBS wash ( $15 \mathrm{~min})$, the polymerase reaction mixture $(0.0625$ $\mathrm{U} / \mathrm{ml}$ of polymerase enzyme (from E .Coli, Klenow Fragment, purchased from NEB)) was added, see Fig. 1 (step c). Under the assumption of ideal reaction conditions, the given amount of enzyme should convert all dNTPs contained in the reaction mixture within a few minutes. However, we extended this reaction step for $2.5 \mathrm{~h}$ to investigate sec ondary remodeling processes. Finally, another threefold PBS wash was performed in order to remove non specifically bound reactants and the remaining enzyme complexes.

\subsection{Fabrication of LSPR substrates}

The fabrication of LSPR gold nanostructures was based on a well established three step process consisting of gold deposition, de wetting and glass etching (Bhalla et al., 2018b). Briefly, a $4 \mathrm{~nm}$ gold film was evaporated on a silicon wafer coated with $500 \mathrm{~nm}$ of $\mathrm{SiO}_{2}$ (KST, Japan) using an electron beam evaporator (MEB550S2 HV, PLASSYS Bestek, France). The film was then annealed at $560{ }^{\circ} \mathrm{C}$ for $3.5 \mathrm{~h}$, forming in dividual gold islands due to solid state de wetting of the gold film (see Fig. 2 a d). These nanoislands were transformed to pillar like nanos tructures with $\mathrm{SiO}_{2}$ stems and Au caps by selective etching of the $\mathrm{SiO}_{2}$ layer. Reactive ion $\mathrm{SF}_{6}$ plasma was applied using an inductively coupled plasma chemical vapor deposition equipment (Plasmalab 100, Oxford Instruments, UK).

\subsection{Characterization of LSPR substrates}

Scanning electron microscopy (SEM) was used to characterize the size and morphology of the Au nanostructures. The average diameter and cap to cap distance were obtained by using the particle analysis module in ImageJ software (Schindelin et al., 2012). The Au caps were assumed to be circular and bright in the image with threshold type processing. The detailed morphology of Au nanostructures were ana lyzed after applying a contrast threshold with three independent images.

\subsection{Fabrication of microfluidic chips with LSPR substrates}

The microfluidic LSPR chip involves three layered substrates: the LSPR Si substrate containing Au plasmonic nanostructures, a trans parent Polydimethylsiloxane (PDMS) layer, and a transparent poly (methyl methacrylate) (PMMA) layer. To ensure tight bonding between the LSPR substrate and PDMS, the Si wafer $(2 \times 4 \mathrm{~cm})$ was covered by a mask with open circles of $5 \mathrm{~mm}$ in diameter. This ensures that $\mathrm{Au}$ na nostructures were fabricated only inside the circular areas during the Au evaporation, annealing and etching steps. The PDMS containing a central circular reaction area of $19.6 \mathrm{~mm}^{2}$ was then bonded with the LSPR substrate by using oxygen plasma. On top of the PDMS layer, a poly methyl methacrylate (PMMA) cuboid $(25 \times 15 \times 8 \mathrm{~mm})$ with a cy lindrical hole ( $8 \mathrm{~mm}$ in diameter) was attached by using a double sided tape. This PMMA layer served as a water reservoir for indentation of the fibre optics, consisting of the LSPR light source and the detector (see detailed schematic in Fig. 2 e f). The inlet of the PDMS channel was connected to the tubing system using a connector needle. To introduce new reactants and carry out the necessary washing steps, fluids were withdrawn with a syringe pump at a flow rate of $50 \mu \mathrm{l} / \mathrm{min}$. This flow rate avoided bubble formation and enabled stable flow in the micro fluidic chip.

\subsection{LSPR measurements on bare nanoplasmonic substrates}

A customized setup consisting of a stage, a spectrometer (USB4000 UV VIS ES, Ocean Optics, Japan), a combined light source and de tecting probe (Ocean Optics, Japan) and an optical fiber (Ocean Optics, Japan) connecting the latter was assembled to measure light reflected by the nanoplasmonic structures. Prior to each measurement, bright and dark reference spectra were recorded using a custom matlab rou tine developed in our lab. This allowed the automatic calculation of maximum wavelength and peak shifts from the LSPR in the Au nanos tructures. After an initial reflection measurement of the bare LSPR substrate, the whole reaction was performed as described in sections 2.1 and 2.2. Briefly, $80 \mu \mathrm{l}$ of template and spacer solution were poured into the PMMA well fixed on the nanostructured LSPR substrate and after $16 \mathrm{~h}$ of immobilization, primer binding and polymerase reaction was performed. After the last PBS washing step, the LSPR signal of the functionalized chip was measured. For each of the conditions, at least three LSPR substrates were used for measurements and shifts of the absorption maximum $\Delta \lambda$ were calculated by subtracting the initial maximum wavelength of each individual LSPR substrate $\lambda_{\text {blank }}$. To avoid salt residues, we decreased the PBS concentration of the washing solution step wise and finally washed it with de ionized water. After drying with compressed air, LSPR signals were measured.

For the characterization of the refractive index sensitivity, freshly prepared bare LSPR substrates were used. Water $(\mathrm{RI}=1.333)$, acetone $(\mathrm{RI}=1.356)$, isopropanol $(\mathrm{RI}=1.376)$, mineral oil $(\mathrm{RI}=1.466)$, and toluene $(\mathrm{RI}=1.496)$ were poured into the cylindrical well and the wavelength spectrum of the reflected light was measured while the probe was indented into the solvents. The sensitivity was calculated as the slope of the linear regression of the wavelength maximum $\lambda_{\max }$ plotted over the solvents' refractive index RI. The refractive index re ference values were measured at room temperature using a spectro photometer (UV Vis 1800, Shimadzu, Japan) and compared to litera ture values.

\subsection{Real time microfluidic LSPR measurements}

In real time measurements, the developed LSPR microfluidic chip (see Fig. 2 e f) was used at room temperature. The washing liquids and reaction mixtures were introduced through the inlet reservoir and withdrawn by a syringe pump. The spectrum was recorded con tinuously every $15 \mathrm{~s}$ during the entire duration of the experiment $(\sim 20 \mathrm{~h})$. The wavelength shifts were captured at the end of each reac tion step, presented as the mean value with standard deviation based on at least three independent experiments. The microfluidic setup has a closed fluid loop to prevent solvent evaporation.

\section{Results and discussion}

\subsection{Characterization of bare LSPR substrates for the detection of DNA polymerase reaction}

The sensitivity of the nanoplasmonic substrate was first verified by using different solvents with known refractive indices (RI) in the re levant range for DNA monolayers (i.e., $\mathrm{RI}_{\mathrm{ssDNA}} \sim 1.45$ and $\mathrm{RI}_{\mathrm{dsDNA}} \sim 1.52$ (Elhadj et al., 2004)). Fig. 3 a shows a linear fit $\left(\mathrm{R}^{2}=0.95\right)$ of wave length shifts versus RI with a slope of $54 \pm 6 \mathrm{~nm} / \mathrm{RIU}$. This slope is essentially the RI sensitivity of the nanoplasmonic substrate in the range of refractive indices of ssDNA and dsDNA. In addition, we require a minimum of $0.0625 \mathrm{U} / \mathrm{ml}$ of polymerase to see changes in LSPR signal and therefore we consider this value as the limit of detection of our sensor. Resulting LSPR spectra from polymerase reaction are shown in Fig. $3 \mathrm{~b}$ and mean values of three independent experiments are sum marized in Fig. 3 c. These values were calculated as shifts between the bare LSPR substrate and the LSPR substrate with double stranded DNA after the whole polymerase reaction was completed. 


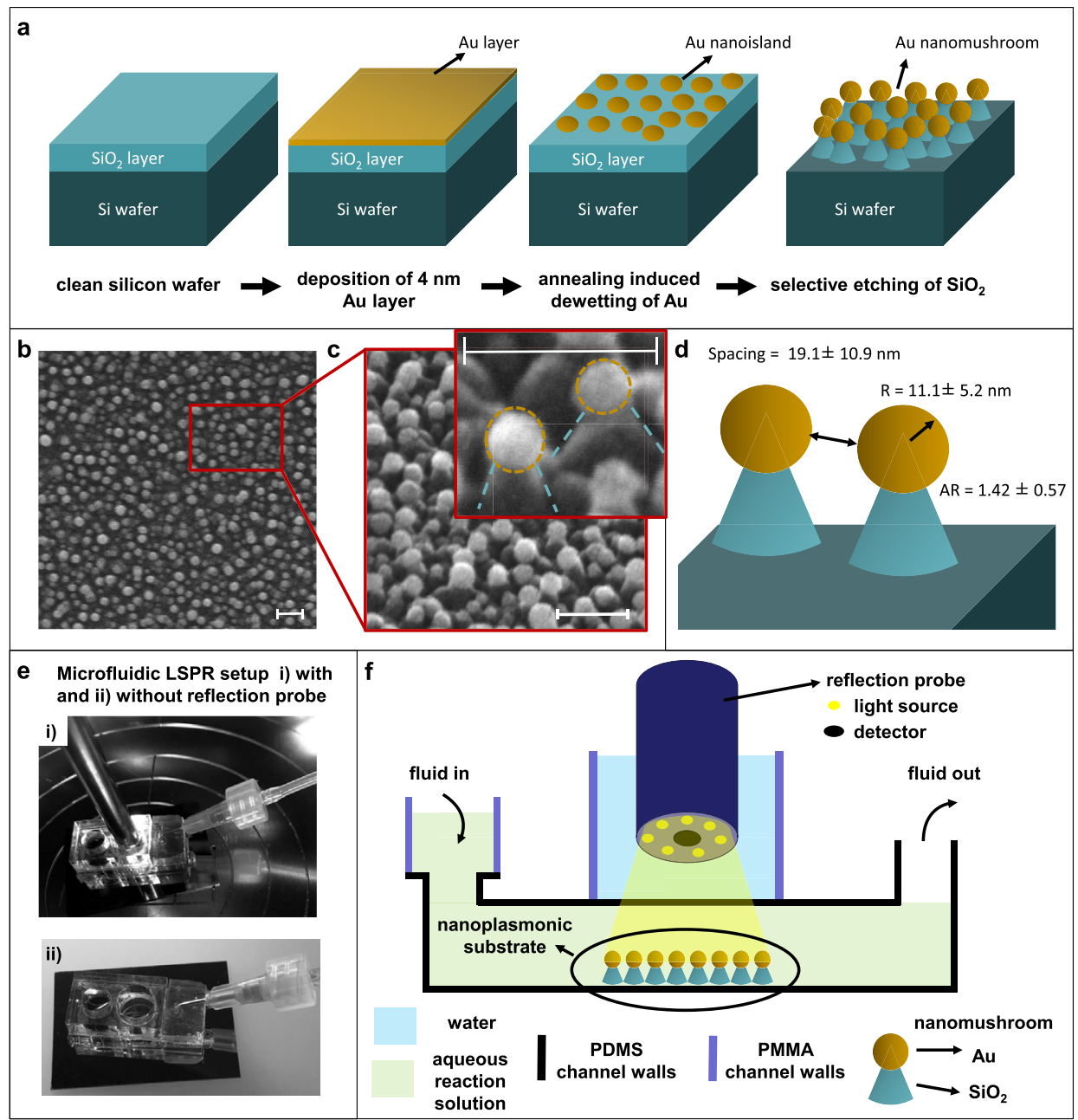

Fig. 2. Fabrication of LSPR-microfluidic platform. (a) Manufacturing of plasmonic surfaces starting from a bare silicon wafer on which a $4 \mathrm{~nm}$ gold layer is first deposited, thermally de-wetted before the $\mathrm{SiO}_{2}$ layer is selectively etched using $\mathrm{SF}_{6}$ plasma. (b) Scanning electron microscopy (SEM) images show the Au nanostructures in horizontal plane, top view, (c) side view with $40^{\circ}$ tilted, with the inset showing the zoomed in view of two pillared nanostructures with the gold cap and $\mathrm{SiO}_{2}$ stem, outlined in yellow and turquoise, respectively. All scale bars represent $100 \mathrm{~nm}$. (d) Schematic of the inset in (c) showing the detailed dimensions of the nanopillar structures. The mean Au cap radius is $\sim 11.1 \pm 5.2 \mathrm{~nm}$. (e) Snap shots of a LSPR-microfluidic chip, in operation with indented reflection probe (i) and without (ii). In both cases the fluid inlet reservoir and the outlet tubing are shown. (f) Schematic of the microfluidic nanoplasmonic chip consisting of the bottom nanoplasmonic substrate, a PDMS and a poly(methyl methacrylate) (PMMA) substrate. (For interpretation of the references to colour in this figure legend, the reader is referred to the Web version of this article.)

Based on the information shown in Fig. 3 a, the theoretical shift caused by the polymerization of double stranded DNA, $\Delta(\mathrm{RI})=0.06$ corresponds to $\Delta \lambda \sim 3.24 \mathrm{~nm}$. In our DNA polymerase experiment (see condition (E) in Fig. 3 c), a shift of $4.19 \pm 0.48 \mathrm{~nm}$ was obtained. This shift represents both the immobilization of ssDNA/HDT and the poly merase reaction. In the control experiments without the polymerase enzyme (C, control without enzyme), a mean shift of $\Delta \lambda=1.66 \pm 2.81$ $\mathrm{nm}$ was observed (see Fig. $3 \mathrm{c}$ and d). Note that the immobilization of ssDNA/HDT alone causes a shift of $3.50 \pm 1.27 \mathrm{~nm}$, which was measured after the immobilization process and the subsequent washing and drying of the LSPR substrate with compressed air. These values were calculated by normalization of wavelength shifts with respect to the blank LSPR substrate prior to the start of the experiment. In contrast, in the control experiment without dNTPs (I, enzyme inhibition), obtained wavelength shifts $(\Delta \lambda=5.66 \pm 1.80 \mathrm{~nm})$ were much higher. One po tential explanation is that after polymerase molecules attach to the ssDNA, these molecules cannot be released from the DNA strand during the washing steps. This increases the local optical density on the sensor surface, which in turn causes an additional red shift. Most importantly, in order to avoid effects of the liquid meniscus in the light path, the actual wavelength shifts need to be evaluated while immersing the probe (see measurement of RIs of different solvents) or after drying the LSPR surfaces with compressed air. The drying of the substrate can precipitate salts from the buffer solution, which might remain on the nanostructures of the LSPR substrate, leading to larger LSPR shifts. This can affect the refractive index on the LSPR substrate, which may lead to poor reproducibility of the LSPR measurements. An immediate wash with DI water avoids the salt precipitation from buffer solution. How ever, the DNA/HDT self assembled monolayer (SAM) optical density and/or functionality might be affected by the inappropriate buffer condition, which can cause indistinguishable LSPR shifts among ex periments and controls. An improvement in the combination of these two processing steps (drying to avoid meniscus and washing with DI water) can enhance the specificity in the LSPR measurements and en sure the bio functionality for subsequent reaction steps. In the next section we show that the use of microfluidics can eliminate many of the issues raised above by controlling the fluid in an automated manner.

\subsection{LSPR microfluidic chip for real time monitoring of DNA immobilization} and polymerase activity

Incorporating nanoplasmonic substrates in a microfluidic system 

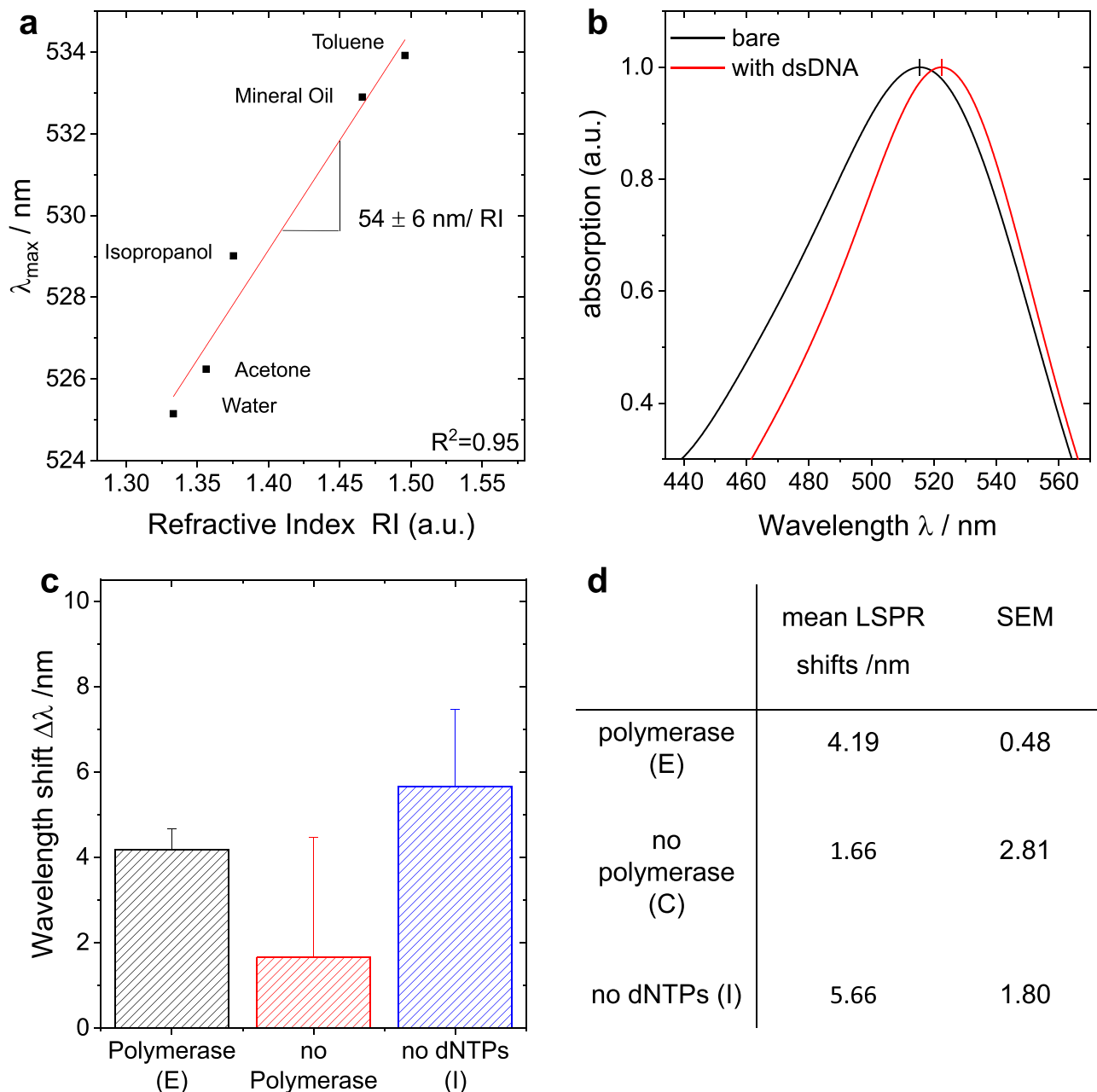

d

\begin{tabular}{l|cc} 
& $\begin{array}{c}\text { mean LSPR } \\
\text { shifts } / \mathrm{nm}\end{array}$ & SEM \\
\hline $\begin{array}{c}\text { polymerase } \\
(\mathrm{E})\end{array}$ & 4.19 & 0.48 \\
$\begin{array}{c}\text { no } \\
\text { polymerase } \\
(\mathrm{C})\end{array}$ & 1.66 & 2.81 \\
$\begin{array}{c}\text { no dNTPs (I) } \\
\text { no dNT }\end{array}$ & 5.66 & 1.80
\end{tabular}

(C)

Fig. 3. DNA polymerase monitoring using discontinuous LSPR measurements. (a) refractive index sensitivity of the nanoplasmonic substrate in a relevant RI range for DNA layers, calculated by linear regression from LSPR measurements with five different solvents; (b) A typical absorption spectrum of a bare nanoplasmonic substrate and after completing immobilization and elongation of ds30-mers (normalized), showing a wavelength shift $\Delta \lambda=3.8 \mathrm{~nm}$; (c) resulting shifts after completing the whole reaction cycle of the polymerase experiment (E, black), control without enzyme (C, red) and substrate inhibition (I, blue), shown as the mean values of $\mathrm{N}=3$ experiments. (d) Table summarizing the values in subfigure (c). (For interpretation of the references to colour in this figure legend, the reader is referred to the Web version of this article.)

allowed real time measurements of complete ssDNA/HDT immobiliza tion and polymerization reaction steps. An exemplary sensogram of our LSPR experiment is shown in Fig. 4 a where LSPR wavelength shifts relative to the functionalized chip (PBS wash after immobilization) are plotted. Note that the response time of our LSPR sensor is $1 \mathrm{~s}$. However, this sensor response time is tunable with software where the data was acquired every $15 \mathrm{~s}$ during the $20 \mathrm{~h}$ real time measurement. The ac quisition time then defines the response time to ensure that there is no overload of the data in the hard drive of our in lab measurement system. Fig. 4b compares the total red shifts in the LSPR signal of a bare LSPR/microfluidic chip in PBS and dsDNA after polymerization reac tion. It is possible to track the continuous red shifts in the LSPR wa velength maximum during the first $12 \mathrm{~h}$ of the ssDNA/HDT im mobilization process. After $12 \mathrm{~h}$, the LSPR signal starts to stabilize and saturation was achieved at $16 \mathrm{~h}$, which was considered as the end of the ssDNA/HDT immobilization. In the following primer binding and washing steps, around $\sim 1.49 \mathrm{~nm}$ shifts were observed. After addition of polymerase, a shift of $\sim 1.1 \mathrm{~nm}$ was detected. This was most likely caused by the binding of the enzyme at the DNA strands and by the binding of additional dNTPs to the DNA strand. After the first $15 \mathrm{~min}$ of the elongation period, a small wavelength shift $(\sim 0.5 \mathrm{~nm})$ was observed. This time scale fits well with the theoretical reaction speed of 0.25 units of enzyme per reaction $(0.0625 \mathrm{U} / \mathrm{ml})$ that are estimated to react with all the available dNTPs ( $10 \mu$ moles) within 16 min. It should be noted that only a small fraction of the available dNTPs can be bound to the immobilized template, thus the elongation reaction completed much sooner than $16 \mathrm{~min}$, which in turn serves as an explanation for the stabilization of the LSPR signal during the remaining elongation time. At the end of the reaction and the final washing step, the release of the heavy enzyme molecules caused a blue shift of $1.2 \mathrm{~nm}$. In the control experiment (C) without polymerase enzyme, varied amounts of LSPR shifts occurred after the reaction was accomplished. This is attributed to various amounts of non specifically attached dNTPs in between ad jacent DNA molecules. The non specific attachment creates a large standard deviation in this control experiment (see Fig. 4c), resulting in low significance of this data as compared to the polymerase reaction ( $\mathrm{p}=0.1744$, unpaired one tailed $t$ test). However, this non specific attachment of dNTPs could be reduced by changing the spacing be tween ssDNA molecules by varying the ratio of DNA/HDT in the first step of the experiment. Despite different amounts of non specific at tachment of dNTPs, the polymerase reaction (E, black curve in Fig. 4a) and the control without enzyme (C, red curve in Fig. 4a) can easily be distinguished in real time.

Moreover, in both control and experimental conditions, no 


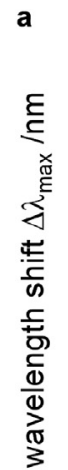

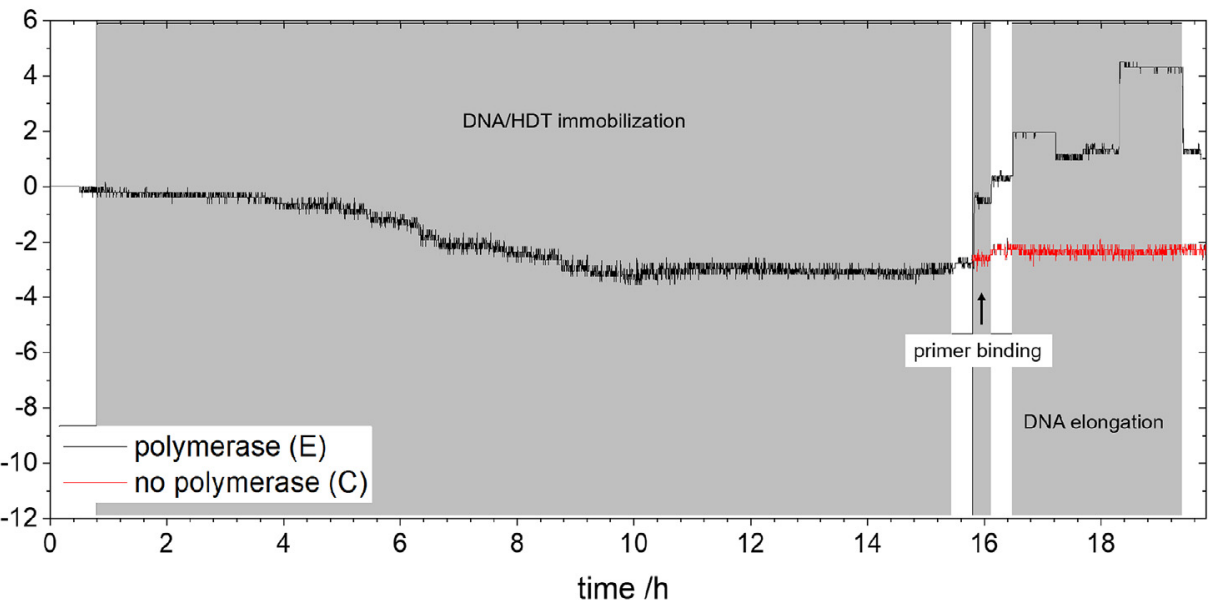

b

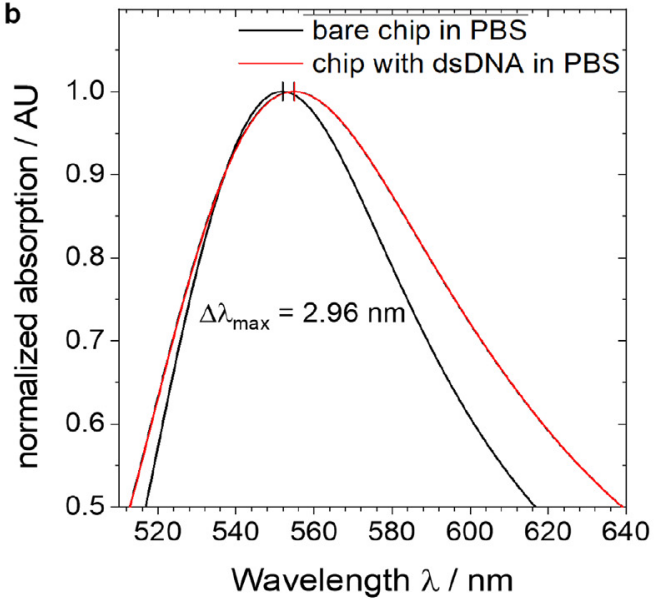

c

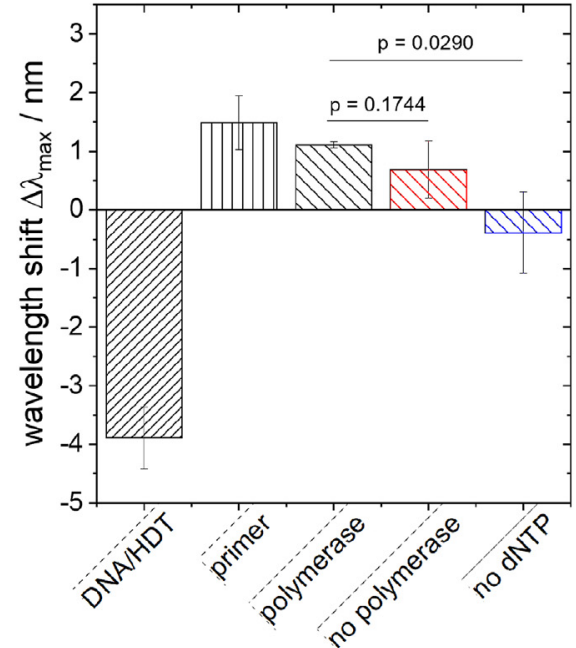

Fig. 4. Label-free real-time DNA/HDT immobilization and polymerase activity monitoring using LSPR measurements. (a) Realtime sensogram showing the shift in the maximum wavelength of the reflected light during immobilization of DNA and HDT, primer binding, DNA elongation and intermediate washing steps. (b) A sample reflection spectra of bare microfluidic chip and the chip with ds30-mer showing a total wavelength shift of $2.7 \mathrm{~nm}$, (c) and mean wavelength shifts from each step, calculated from 6 polymerase reactions and 3 controls (no polymerase and no dNTPs) experiments, respectively. Error bars represent standard error of mean. The polymerase versus "no dNTP" is significant with $\mathrm{p}<0.05$. significant wavelength shifts were detected due to the change of buffer solutions, indicating that the buffer effects can be neglected in these LSPR experiments (Diéguez et al., 2009). This is crucial for comparison of individual steps in a continuous reaction inside the microfluidic chip (where fluid control is automated) which often requires different buffer solutions for biochemical reasons. A total shift of $\Delta \lambda_{\max }=2.96 \mathrm{~nm}$ in the LSPR maximum wavelength was observed after polymerization re action was completed (see Fig. 4 b). An experimental cycle consists of the relative shifts during ssDNA/HDT immobilization (mean of $-3.89 \pm 0.64 \mathrm{~nm}$ ), primer binding (mean of $1.49 \pm 0.46 \mathrm{~nm}$ ) and elon gation (mean of $1.11 \pm 0.06 \mathrm{~nm}$ ). Normalize by the wavelength from the functionalized chip in PBS (step 3), the mean values of all the shifts are summarized in Fig. $4 \mathrm{c}$. The most obvious shifts were obtained during ssDNA/HDT immobilization and elongation steps, whereas during primer binding only one significant shift occurred.

In contrast, the positive control condition with no dNTPs, leads to a slight blue shift of $-0.39 \pm 0.98 \mathrm{~nm}$. This is due to the specific binding of polymerase which is expected as no elongation takes place and the polymerase enzyme has no chance to be released from the ssDNA. However, standard one tailed, $t$ test reveals that this experiment is significant when compared to the polymerase reaction as the value $\mathrm{p}=0.0290$. This also shows that with the use of microfluidics, certain amount of non specific attachment due to inefficient washing in dis continuous LSPR measurements (as seen from Fig. 3) can be minimized.

To validate the results from the microfluidic LSPR sensing system we also used QCM D to monitor all the steps involved in the polymerase reaction. Fig. 5a shows both the frequency (black curve) and dissipation (blue curve) changes in real time caused by immobilization of ssDNA and subsequent elongation of dsDNA strands upon completion of the aforementioned reaction steps. Fig. 5b displays the shifts in the fre quency for each step involved in the reaction and Fig. 5c shows the quantitative analysis of QCM D where frequency shifts are correlated with the molecular weight of the mass bound on the surface of the QCM D. Fig. 5b illustrates that the shifts upon primer binding cannot be distinguished from PBS wash as minute mass changes upon binding of primer is masked by the bulk effects from the buffer. Nevertheless, the QCM D results suggest that the wavelength shifts in the LSPR are true signatures of the polymerase activity. More details on the QCM D measurement principles and discussion on Fig. 5 can be found in the supplementary information.

\section{Conclusion}

We demonstrated the use of nanoplasmonic LSPR technology cou pled with microfluidics to monitor the formation of SAMs of ssDNA, and subsequently detect the interaction of DNA with the DNA polymerase enzyme, in real time and label free manner. The nanoplasmonic struc tures, fabricated by thermal de wetting and reactive ion etching of Au, possessed a RI sensitivity of $54 \pm 6 \mathrm{~nm} / \mathrm{RIU}$ in the relevant range of refractive indices of single and double stranded DNA. The LSPR results for monitoring ssDNA/HDT immobilization and the polymerase reac tion were validated by using QCM D in real time. Both sensing meth odologies, LSPR and QCM D, suggested that surface functionalization with ssDNA T30 took approximately $12 \mathrm{~h}$, which is in good accordance with the typical protocols proposing a reaction time of $1216 \mathrm{~h}$. Our work showed that the self assembly of biochemical monolayers, char acterization of enzyme kinetics and inhibition reactions under physio logical conditions could now be tested by using labe free LSPR in real 


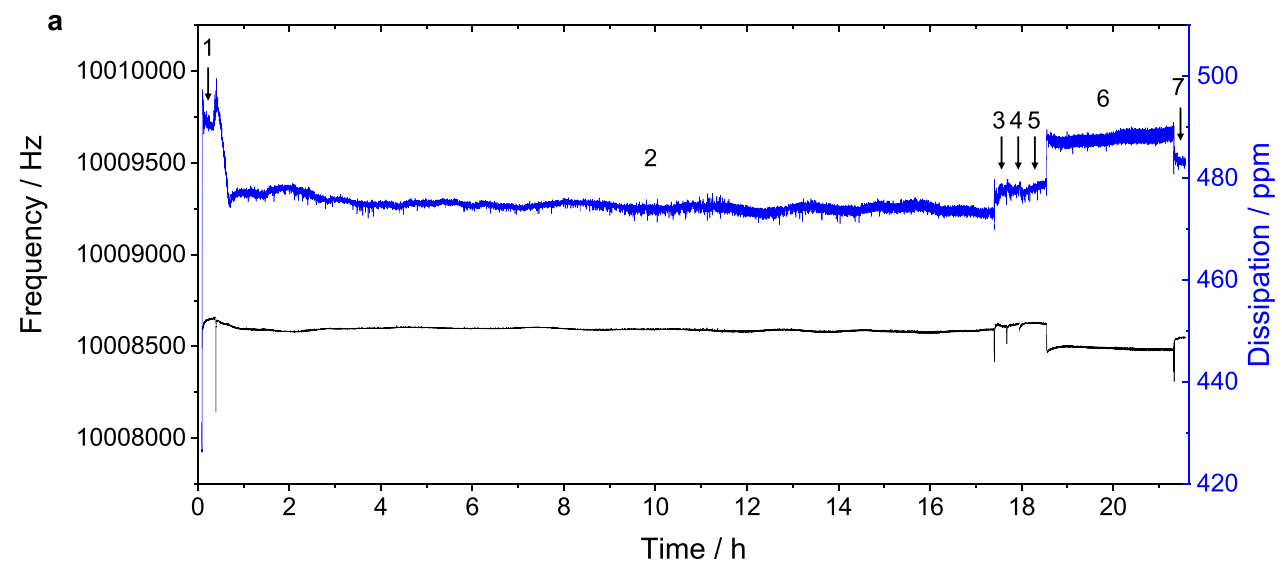

b

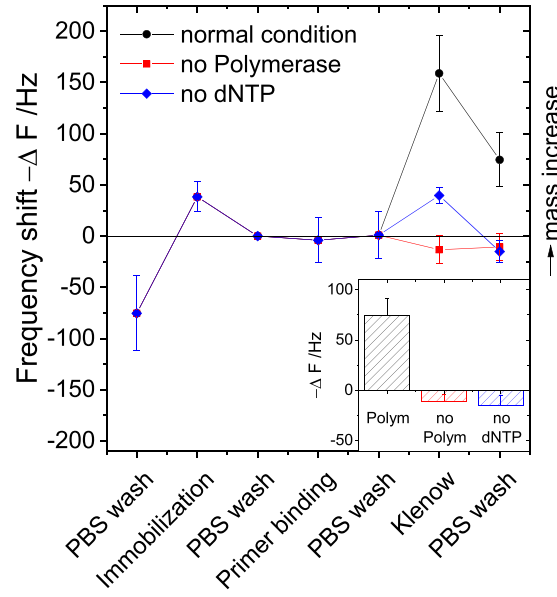

Fig. 5. DNA polymerase monitoring with QCM-D. (a) Real sensogram showing the temporal course of frequency (black) and dissipation (blue) during immobilization of DNA (2); primer binding (4), DNA elongation (6) and all corresponding washing steps $(1,3,5,7)$. (b) Frequency shifts during the aforementioned reaction steps of the polymerase reaction (E, black circles), control without enzyme (C, red squares) and substrate inhibition (I, blue diamonds), results from $\mathrm{N} \geq 3$ independent experiments, shown as mean and standard deviation. In the inset, the frequency shift during the crucial elongation step is highlighted. It was calculated as shift from washing before elongation to washing after elongation. (c) Proof of quantitativeness of QCM-D sensing by correlating the step-wise shifts, acquired at the end of each washing step (in PBS buffer) with the molecular weight that is theoretically bound during the corresponding step. Values are normalized to the molecular weight of T30 ( 9190 g/mol). More details can be found in part 1 of the supplementary information file. (For interpretation of the references to colour in this figure legend, the reader is referred to the Web version of this article.) time with limited human intervention during the course of the reaction. These features are of great interest for the development of nanobio sensors for biomedical applications. Some limitations of our current platform include the lack of temperature control in the microfluidic chip and the need to optimize the HDT/ssDNA surface chemistry to reduce the non specific attachment of dNTP without polymerase en zyme. However, the architecture of the microfluidic chip and the LSPR measurement in the reflection mode allow easy integration of tem perature controller in the future. As the polymerase reaction serves as the backbone of DNA sequencing, our LSPR microfluidic chip can also benefit from the integration of a portable LSPR readout for point of care sequencing applications in the future. Therefore our LSPR microfluidic platform serves as a benchmark system for emerging fields in clinical, pharmaceutical and scientific research which require efficient, easy to use, precise methods for comprehensive data collection.

\section{Acknowledgements}

Authors would like to thank Mr. Hung Ju Chiang from Okinawa Institute of Science and Technology Graduate University (OIST) for providing help in DNA sample preparations. All authors would also like to acknowledge the support of OIST with subsidy funding from the Cabinet Office, Government of Japan. AQS also acknowledges financial support from the Japanese Society for the Promotion of Science under grants 17K06173 and 18H01135. KYC and NB also acknowledge the support by the OIST Technology Development and Innovation Center's Proof of Concept Program.

\section{References}

Aćimović, S.S., Ortega, M.A., Sanz, V., Berthelot, J., Garcia-Cordero, J.L., Renger, J., Maerkl, S.J., Kreuzer, M.P., Quidant, R., 2014. Lspr chip for parallel, rapid, and sensitive detection of cancer markers in serum. Nano Lett. 14 (5), 2636-2641.

Anker, J.N., Hall, W.P., Lyandres, O., Shah, N.C., Zhao, J., Van Duyne, R.P., 2010. Biosensing with plasmonic nanosensors. In: Nanoscience and Technology: A Collection of Reviews from Nature Journals. World Scientific, pp. 308-319.

Baaske, M.D., Foreman, M.R., Vollmer, F., 2014. Single-molecule nucleic acid interactions monitored on a label-free microcavity biosensor platform. Nat. Nanotechnol. 9 (11), 933-939.

Benkovic, S.J., Cameron, C.E., 1995. [20] kinetic analysis of nucleotide incorporation and misincorporation by klenow fragment of escherichia coli dna polymerase i. In: Campbell, J.L. (Ed.), DNA Replication. Vol. 262 of Methods in Enzymology, 00766879. Academic, San Diego, Calif. and London, pp. 257-269.

Bhalla, N., Chiang, H.-J., Shen, A.Q., 2018a. Cell biology at the interface of nanobiosensors and microfluidics. Methods Cell Biol. 148, 203-227.

Bhalla, N., Sathish, S., Sinha, A., Shen, A.Q., 2018b. Large-scale nanophotonic structures for long-term monitoring of cell proliferation. Adv. Biosyst. 2 (4), 1700258.

Bingen, P., Wang, G., Steinmetz, N.F., Rodahl, M., Richter, R.P., 2008. Solvation effects in the quartz crystal microbalance with dissipation monitoring response to biomolecular adsorption. a phenomenological approach. Anal. Chem. 80 (23), 8880-8890.

Cheng, X.R., Hau, B.Y.H., Endo, T., Kerman, K., 2014. Au nanoparticle-modified dna sensor based on simultaneous electrochemical impedance spectroscopy and localized surface plasmon resonance. Biosens. Bioelectron. 53, 513-518.

Diéguez, L, Darwish, N., Mir, M., Martínez, E, Moreno, M., Samitier, J., 2009. Effect of the refractive index of buffer solutions in evanescent optical biosensors. Sens. Lett. 7 (5), 851-855.

Elhadj, S., Singh, G., Saraf, R.F., 2004. Optical properties of an immobilized dna monolayer from 255 to $700 \mathrm{~nm}$. Langmuir 20 (13), 5539-5543.

Endo, T., Kerman, K., Nagatani, N., Takamura, Y., Tamiya, E., 2005. Label-free detection of peptide nucleic acid-dna hybridization using localized surface plasmon resonance based optical biosensor. Anal. Chem. 77 (21), 6976-6984.

Griep, M.A., 1995. Fluorescence recovery assay: a continuous assay for processive dna polymerases applied specifically to dna polymerase iii holoenzyme. Anal. Biochem. 232 (2), 180-189.

Haber, J.M., Gascoyne, P.R.C., Sokolov, K., 2017. Rapid real-time recirculating pcr using localized surface plasmon resonance (lspr) and piezo-electric pumping. Lab Chip 17 (16), 2821-2830.

Hammond, J.L., Bhalla, N., Rafiee, S.D., Estrela, P., 2014. Localized surface plasmon resonance as a biosensing platform for developing countries. Biosensors 4 (2), 
$172-188$.

Huang, C., Ye, J., Wang, S., Stakenborg, T., Lagae, L., 2012. Gold nanoring as a sensitive plasmonic biosensor for on-chip dna detection. Appl. Phys. Lett. 100 (17), 173114.

Kaye, S., Zeng, Z., Sanders, M., Chittur, K., Koelle, P.M., Lindquist, R., Manne, U., Lin, Y., Wei, J., 2017. Label-free detection of dna hybridization with a compact lspr-based fiber-optic sensor. The Analyst 142 (11), 1974-1981.

Kim, E., Baaske, M.D., Schuldes, I., Wilsch, P.S., Vollmer, F., 2017. Label-free optical detection of single enzyme-reactant reactions and associated conformational changes. Sci. Adv. 3 (3), e1603044.

Luka, G., Ahmadi, A., Najjaran, H., Alocilja, E., DeRosa, M., Wolthers, K., Malki, A., Aziz, H., Althani, A., Hoorfar, M., 2015. Microfluidics integrated biosensors: a leading technology towards lab-on-a-chip and sensing applications. Sensors (Basel, Switzerland) 15 (12), 30011-30031.

Matsuno, H., Niikura, K., Okahata, Y., 2001. Direct monitoring kinetic studies of dna polymerase reactions on a dna-immobilized quartz-crystal microbalance. Chem. Eur J. 7 (15), 3305-3312.

Mayer, K.M., Hafner, J.H., 2011. Localized surface plasmon resonance sensors. Chem. Rev. 111 (6), 3828-3857.

Oh, B.-R., Huang, N.-T., Chen, W., Seo, J.H., Chen, P., Cornell, T.T., Shanley, T.P., Fu, J., Kurabayashi, K., 2014. Integrated nanoplasmonic sensing for cellular functional immunoanalysis using human blood. ACS Nano 8 (3), 2667-2676.

Park, K.H., Kim, S., Yang, S.-M., Park, H.G., 2009. Detection of dna immobilization and hybridization on gold/silver nanostructures using localized surface plasmon resonance. J. Nanosci. Nanotechnol. 9 (2), 1374-1378.

Ronaghi, M., 2001. Pyrosequencing sheds light on dna sequencing. Genome Res. 11 (1),
3-11.

Sannomiya, T., Hafner, C., Voros, J., 2008. In situ sensing of single binding events by localized surface plasmon resonance. Nano Lett. 8 (10), 3450-3455.

Schindelin, J., Arganda-Carreras, I., Frise, E., Kaynig, V., Longair, M., Pietzsch, T., Preibisch, S., Rueden, C., Saalfeld, S., Schmid, B., Tinevez, J.-Y., White, D.J., Hartenstein, V., Eliceiri, K., Tomancak, P., Cardona, A., 2012. Fiji: an open-source platform for biological-image analysis. Nat. Methods 9 (7), 676-682.

Schneider, T., Jahr, N., Jatschka, J., Csaki, A., Stranik, O., Fritzsche, W., 2013. Localized surface plasmon resonance (lspr) study of dna hybridization at single nanoparticle transducers. J. Nanoparticle Res. 15 (4), 442.

Seville, M., West, A.B., Cull, M.G., McHenry, C.S., 1996. Fluorometric assay for dna polymerases and reverse transcriptase. Biotechniques 21 (4) 664, 666, 668, 670, 672.

Shapiro, A., Rivin, O., Gao, N., Hajec, L., 2005. A homogeneous, high-throughput fluorescence resonance energy transfer-based dna polymerase assay. Anal. Biochem. 347 (2), 254-261.

Soares, L., Csáki, A., Jatschka, J., Fritzsche, W., Flores, O., Franco, R., Pereira, E., 2014. Localized surface plasmon resonance (lspr) biosensing using gold nanotriangles: detection of dna hybridization events at room temperature. The Analyst 139 (19), 4964-4973.

Szunerits, S., Boukherroub, R., 2012. Sensing using localised surface plasmon resonance sensors. Chem. Commun. 48 (72), 8999-9010.

Touahir, L., Galopin, E., Boukherroub, R., Gouget-Laemmel, A.C., Chazalviel, J.-N., Ozanam, F., Szunerits, S., 2010. Localized surface plasmon-enhanced fluorescence spectroscopy for highly-sensitive real-time detection of dna hybridization. Biosens. Bioelectron. 25 (12), 2579-2585. 
Karlsruher Institut für Technologie

\section{Repository KITopen}

Dies ist ein Postprint/begutachtetes Manuskript.

Empfohlene Zitierung:

Roether, J.; Chu, K.-Y.; Willenbacher, N.; Shen, A. Q.; Bhalla, N.

Real-time monitoring of DNA immobilization and detection of DNA polymerase activity by a microfluidic nanoplasmonic platform.

2019. Biosensors and bioelectronics, 142.

doi: $\underline{10.5445 / / R / 1000097089}$

Zitierung der Originalveröffentlichung:

Roether, J.; Chu, K.-Y.; Willenbacher, N.; Shen, A. Q.; Bhalla, N.

Real-time monitoring of DNA immobilization and detection of DNA polymerase activity by a microfluidic nanoplasmonic platform.

2019. Biosensors and bioelectronics, 142, Art.-Nr.: 111528.

doi:10.1016/j.bios.2019.111528

Lizenzinformationen: CC BY NC ND 4.0-Lizenz 